\title{
tic\&société
}

Vol. 15, $\mathbf{N}^{\circ} 1 \mid$ | 1 er semestre $2021 \mid 2021$

Logique algorithmique et reproduction sociétale: les médiations sociales saisies par les algorithmes

Langage, post-politique et automatisation : critique préventive de

l'argumentation artificielle

Language, the Post-political and Automation: A Preventive Critique of Artificial Argumentation

Lenguaje, post-política y automatización : crítica preventiva de la argumentación artificial

\section{Samuel COSSETTE}

\section{OpenEdition}

Journals

Édition électronique

URL : https://journals.openedition.org/ticetsociete/5764

DOI : 10.4000/ticetsociete.5764

Éditeur

Association ARTIC

Édition imprimée

Pagination : 69-96

\section{Référence électronique}

Samuel COSSETTE, "Langage, post-politique et automatisation : critique préventive de l'argumentation artificielle 》, tic\&société [En ligne], Vol. 15, N 1 | 1er semestre 2021 | 2021, mis en ligne le 20 mai 2021, consulté le 22 mai 2021 URL : http://journals.openedition.org/ticetsociete/5764; DOI : https://doi.org/10.4000/ticetsociete. 5764 
Langage, post-politique et automatisation : critique

préventive de l'argumentation artificielle

SAMUEL COSSETTE

cossette.samuel@courrier.uqam.ca 


\section{Langage, post-politique et automatisation : critique préventive de l'argumentation artificielle}

Samuel Cossette est candidat au doctorat en communication à I'Université du Québec à Montréal (UQAM) et membre du Groupe de recherche sur l'information et la surveillance au quotidien (GRISQ). II étudie principalement les procédés d'automatisation du discours politique, notamment les méthodes de microciblage béhavioral et d'analyse psychographique.

Résumé : Cet article met en discussion les concepts de postpolitique, de gouvernementalité algorithmique et de langage en traçant un portrait des recherches récentes dans le champ de l'argumentation mining, un champ de recherche en Big Data et en linguistique computationnelle. II montre que les recherches en argumentation mining portent un intérêt particulier au débat politique, et offrent des méthodes qui participent au processus de dépolitisation du débat, mais sont encore loin de pouvoir saisir toutes les spécificités du langage.

Mots-clés: post-politique, gouvernementalité algorithmique, langage, débat, minage d'arguments

\section{Language, the post-political and automation: a preventive critique of artificial argumentation}

Abstract: This article discusses the concepts of post-political, algorithmic governance and language in relation to recent research in argumentation mining, a field of study within Big Data and computational linguistics. This article shows that studies in argumentation mining are particularly oriented towards political debate and offer methods to depoliticize the debate process, but are still incapable of grasping all of language's specificities.

Keywords: Post-political, algorithmic governance, language, debate, argumentation mining.

\section{Lenguaje, post-política y automatización : crítica preventiva de la argumentación artificial}

Resumen: Este artículo analiza los conceptos de post-política, gubernamentalidad algorítmica y de lenguaje, estableciendo un panorama de las investigaciones recientes en el campo de la 
minería de argumentación, un campo de investigación que relaciona Big Data y lingüística computacional. Se concluye que las investigaciones en minería de argumentos tienen un interés particular en el debate político a la vez que ofrecen métodos que contribuyen a la despolitización del debate; y que aún están lejos de poder capturar todas las especificidades del lenguaje.

Palabras-clave: Post-política, gubernanza algorítmica, lenguaje, debate, minería de argumentos. 
Les développements technologiques de grande envergure apportent généralement des élans d'imagination (Besnier, 2013). Avec le numérique, et particulièrement le Big Data, c'est entre autres l'imaginaire de la prise de décision automatisée qui s'est imposé, puisque la récolte automatisée de vastes quantités de données porte la promesse de pouvoir directement relier une décision à la réalité "brute ». Présente déjà il y a plusieurs décennies, notamment avec le projet RAND sur l'automatisation des capacités militaires aux États-Unis (Lasswell, 1955), et toujours influente aujourd'hui (Yeung, 2018), l'idée d'automatisation de la prise de décision est attirante pour plusieurs raisons : élimination des erreurs humaines, efficacité de la gestion et fin des longs débats qui caractérisent les processus de prise de décision. Le développement de l'algorithmique et de l'apprentissage profond offre un souffle nouveau à ce rêve aujourd'hui désigné par les chercheurs et chercheuses par l'expression "régulation algorithmique » :

La régulation algorithmique réfère aux systèmes de prise de décision qui régulent un domaine d'activité dans un objectif de gestion des risques ou de modification des comportements, en générant automatiquement et continuellement de nouvelles connaissances grâce à la collecte systématique et continue de données en temps réel. Ces données sont tirées directement d'un ensemble de composantes dynamiques de ce champ d'activité, afin d'identifier et, si nécessaire, de corriger automatiquement des opérations pour atteindre un objectif préalablement spécifiél (Yeung, 2018, p. 505).

$\mathrm{Ce}$ mode de régulation sociale est désigné par Antoinette Rouvroy comme en étant un de "gouvernementalité algorithmique », c'est-à-dire :

un mode de gouvernement nourri essentiellement de données brutes, signaux infra-personnels et a-signifiants mais quantifiables, opérant par configuration anticipative des possibles plutôt que par règlementation des conduites, et ne s'adressant aux individus que par voie d'alertes provoquant

\footnotetext{
1 Citation originale: Algorithmic regulation refers to decision making systems that regulate a domain of activity in order to manage risk or alter behavior through continual computational generation of knowledge by systematically collecting data (in real time on a continuous basis) emitted directly from numerous dynamic components pertaining to the regulated environment in order to identify and, if necessary, automatically refine (or prompt refinement of) the system's operations to attain a pre-specified goal.
} 
des réflexes plutôt qu'en s'appuyant sur leurs capacités d'entendement et de volonté (Rouvroy, 2012, s.p.).

Le concept de gouvernementalité algorithmique permet de bâtir une critique de plusieurs phénomènes communicationnels contemporains. Le phénomène qui nous intéresse est celui du traitement automatisé du langage, plus précisément de l'argumentation automatisée. Dans le cadre de cette courte analyse, nous tenterons d'explorer les liens conceptuels entre le traitement automatisé du langage et la gouvernementalité algorithmique, en mobilisant la notion de " post-politique » et en prenant comme cas d'étude les techniques de " minage d'arguments » (argumentation mining) et d'argumentation automatisée.

\section{Décontextualisation et occultation du politique dans la gouvernementalité algorithmique}

La gouvernementalité algorithmique selon Rouvroy comprend plusieurs dynamiques qui, sans lui être propres, en sont constitutives. Deux de celles-ci nous intéressent particulièrement dans le cadre de cette analyse, soit celles de la décontextualisation (des données, des faits sociaux) et de l'occultation du politique par rapport au sujet, au discours, aux processus décisionnels.

La gouvernementalité algorithmique se déroulerait en trois principales étapes: la récolte et le stockage de données, le traitement des données et la production de connaissances, puis finalement le profilage algorithmique et l'action sur les comportements (Rouvroy et Berns, 2013). Dès la première étape, et en partie dans la seconde, les données sont décontextualisées, coupées du contexte dans lequel elles ont été produites (Bonenfant et al., 2015). Elles sont en ce sens "désaffectées ${ }^{2}$, c'est-à-dire qu'elles ne sont plus affectées (affects) d'une valeur, au sens éthique du terme " (Ouellet et al., 2016, p. 156). Cette décontextualisation est une opération qui est banale et régulière dans les procédures informatiques et statistiques, mais qui n'est toutefois pas sans importance. Dès lors que les données perdent leur référent au social, elles ne deviennent que quantitatives, brutes, et en viennent ainsi à " acquérir les attributs du Réel lui-même " (Ouellet et al., 2016, p. 158).

La décontextualisation des données est donc la première étape de l'occultation du politique: toute prétention d'accéder à un

${ }^{2}$ En italique dans le texte. 
«Réel » brut est en soi une négation du socialement constitué, du politique, dans le sens où les données nous présenteraient quelque chose de naturel, d'indiscutable. De plus, la gouvernementalité algorithmique opère une forme d'occultation supplémentaire dans la troisième étape de son déploiement, soit l'action sur les comportements, qui est une forme de régulation sociale. C'est le passage « d'un monde qui était encore régulé normativement par la culture et qui se reproduisait de manière plus ou moins stabilisé [sic] au moyen d'institutions politiques" à un monde "autonomisé, informatisé et automatisé de régulation de la pratique sociale " (Ouellet et al., 2016, p. 150). Pour Rouvroy (2012, s.p.), il s'agit d'un processus de «dissipation des conditions institutionnelles, spatiales, temporelles et langagières de la subjectivation au profit d'une régulation objective, opérationnelle, des conduites possibles ».

Lorsque Rouvroy parle "d'action sur les comportements », elle désigne le processus à partir duquel, dans la gouvernementalité algorithmique, on cherche à prédire les comportements futurs d'un individu, selon les données récoltées, et on envoie un signal (trigger) pour entraîner le comportement qui a été prédit, ou minimalement l'orienter (Cossette, 2018; Ouellet et al., 2016). C'est là le troisième et dernier niveau de l'occultation menée par la gouvernementalité algorithmique: d'abord sur les données, puis sur le mode de régulation et, finalement, sur le sujet lui-même, qu'on prétend dénué de toute réflexivité. La gouvernementalité algorithmique, en effet :

[transcrit] le monde et ses habitants sous forme de données numériques, métabolisables par les systèmes informatiques. $\mathrm{De}$ cette transcription systématique, a-signifiante, décontextualisante, neutralisante, résultent des masses gigantesques de données brutes, disponibles pour une multitude d'opérations statistiques (Rouvroy, 2012, s.p.). 


\section{Agonisme/antagonisme et débat politique}

Le processus d'occultation du politique présent dans la gouvernementalité algorithmique n'est pas nouveau, mais s'inscrit plutôt dans de plus profonds courants : la cybernétique d'abord, le post-politique ensuite. Tous ces courants partagent une conception commune, soit celle d'une société optimisée et mathématisée, dans laquelle les processus de prises de décision face à un problème sont essentiellement le résultat d'un traitement de l'information disponible - dans son sens large - pour arriver à la solution la plus efficace. Dans la gouvernementalité algorithmique, on ajoute l'attrait de la prédiction et de la prévention, qui permettent de mettre en place des régulations basées sur le risque, une «justice actuarielle " et une organisation sociale systématique basée sur la surveillance (Yeung, 2018, p. 511). De façon globale, on pousse encore plus loin le rêve du post-politique, soit celui d'

une société modélisée comme une entité cybernétique dotée d'une téléologie qui exclue [...] à la base l'antagonisme et la diversité politique. II s'agit d'une société qui, déployée à l'aune du positivisme et de l'empirisme, occulte la synthèse des délibérations propres au politique, c'est-à-dire neutralise les antagonismes politico-idéologiques (la critique) à la base de toute praxis (Ouellet et al., 2016, p. 165).

\subsection{Post-politique}

La notion de post-politique telle qu'elle est comprise aujourd'hui naît essentiellement suite à la chute de I'URSS, au début des années 1990. Elle est incarnée dans la pensée de plusieurs auteurs influents, dont le plus connu (et le plus critiqué) est probablement Francis Fukuyama (1992), avec son livre « The End of History and the Last Man ». Dans son livre, Fukuyama affirme que les démocraties capitalistes libérales constituent l'aboutissement ultime de l'histoire politique de l'humanité, l'ultime mode de gouvernance.

Or, l'expression « la fin de l'histoire » est une métonymie. Ce que Fukuyama entend par là, c'est plutôt la fin du politique : la chute de I'URSS marque pour lui la victoire totale du libéralisme et du capitalisme ainsi que la fin, du même coup, du débat politique. Cette thèse est reprise par Anthony Giddens (1998, p. 44), un autre penseur du "post-politique », qui affirme: "il n'existe pas d'alternative au capitalisme[. L]e seul débat qui reste concerne 
jusqu'où et de quelles manières le capitalisme doit être gouverné et régulé ${ }^{3}$ ". II ne reste qu'à prendre les mesures de bonne gestion et de bonne gouvernance nécessaires pour maintenir cet état de fait : c'est le remplacement du politique par "l'expertise de l'administration sociale ${ }^{4} »($ Zizek, 2005, p. 117).

Le post-politique marquerait donc la fin du débat politique et transformerait le gouvernement en agent de régulation ${ }^{5}$. Ce sont les Third Way politics, qui se nourrissent de la fin effective de l'affrontement entre la gauche socialiste et de la droite capitaliste; les life politics, ni plus ni moins la gestion des problèmes de la vie courante (Giddens, 1994); ou encore les risk politics, un mode de gouvernement actuariel animé par la nécessité de gérer les risques (Beck, 1992).

La gouvernementalité algorithmique selon Rouvroy s'inscrit dans ce contexte, qui serait celui du post-politique. Autant la gouvernementalité algorithmique que dans le post-politique, en effet :

le nouveau critère de validation du savoir ne serait plus la Raison, mais la performativité. Bref, il ne s'agirait plus d'orienter la quête de la connaissance vers un idéal de vérité, mais plutôt en fonction du critère de l'efficience (Ouellet, 2017, p. 4).

\subsection{Agonisme/antagonisme}

L'une des principales détractrices d'Anthony Giddens et des Third Ways Politics, selon lesquels le débat politique serait essentiellement chose du passé, est Chantal Mouffe. Elle est également une influente critique du modèle de démocratie délibérative habermassien. Sa conception de la démocratie et du débat politique, qui s'oppose dans son essence au modèle de gouvernementalité algorithmique - quoique Mouffe n'en parle pas directement -, est intéressante à explorer dans le cadre de notre réflexion. En effet, explique Chantal Mouffe (2010, p. 18-19), « les questions proprement politiques impliquent toujours des décisions

3 Citation originale: There are no alternatives to capitalism[. T]he only argument that remains concerns how far, and in what ways, capitalism should be governed and regulated. ${ }^{4}$ Citation originale: When politics proper is progressively replaced by expert social administration.

${ }^{5}$ II ne faut pas ici tirer la conclusion que le gouvernement n'est plus politique. La régulation sociale se fait dans un cadre bien précis, celui du capitalisme libéral, transformant essentiellement l'État en agent régulateur du libéralisme : c'est le néolibéralisme (Dardot et Laval, 2010), institué dans la « fin du politique ». 
qui exigent de faire un choix entre des alternatives qui sont indécidables d'un point de vue strictement rationnel ». Peut-on alors prétendre qu'une gouvernementalité algorithmique en viendrait à administrer le social de façon entièrement dépolitisée ?

Mouffe distingue dans sa réflexion les concepts d' "agonisme » et d' " antagonisme ». L'antagonisme réfère à un rapport entre ennemis, qui ne peuvent partager de consensus. L'agonisme réfère à un rapport entre adversaires, qui s'affrontent et débattent à l'intérieur d'un certain cadre, d'un certain consensus, que les deux parties respectent. Les adversaires acceptent et reconnaissent ainsi leur présence respective.

Selon Mouffe (2010, p. 19), l'affrontement agonistique est la " condition même d'existence " de la démocratie. Son argument passe par l'introduction du concept d'« hégémonie ». Pour l'autrice, " toute société est le produit de pratiques qui visent à instituer un ordre dans un contexte de contingence. Tout ordre social est donc de nature hégémonique et son origine politique " (Mouffe, 2010, p. 20). Ainsi, le consensus politique (tel qu'il apparaît dans la vision de Giddens, par exemple) est nécessairement hégémonique, qu'il soit atteint grâce à la raison, comme le veut Habermas, ou par l'opinion et la persuasion, comme le veut Arendt.

Toute lutte agonistique suppose selon Mouffe une hégémonie sociale déjà existante (2010). De même, puisque le social est constitué de pratiques hégémoniques, "tout ordre est instauré à travers l'exclusion d'autres possibilités" (Mouffe, 2010, p. 20). L'existence même de ces possibilités forme le politique ${ }^{6}$, qui est la " possibilité toujours présente de l'antagonisme » (2010, p, 20). Les pratiques hégémoniques qui forment le social

occultent les actes originaires de leur institution politique contingente et apparaissent comme si elles procédaient d'un ordre naturel. [...] Tout ordre social qui, à un moment donné, est perçu comme étant «naturel », ainsi que le "sens commun » qui l'accompagne est en fait le résultat de pratiques hégémoniques sédimentées et n'est jamais la manifestation d'une objectivité qui serait extérieure aux pratiques à travers lesquelles il a été établi (Mouffe, 2010, p. 20).

Le social est toujours politique, car il est toujours « l'expression d'une structure particulière de relations de pouvoir " (Mouffe, 2010, p. 20). La critique de Mouffe rejette toute possibilité d'objectivité totale, toute possibilité de consensus parfait, toute possibilité de

${ }^{6}$ En italique dans le texte. 
social dont le politique serait complètement occulté. Pour elle, c'est l'affrontement agonistique, mais surtout antagonistique qui permet d'éviter l'éclipse totale d'une hégémonie. C'est alors la notion de débat qui s'impose comme lieu commun de résistance à une hégémonie sociale totale.

Le débat, donc, est essentiel dans la constitution du politique et du social. Cette constatation est déjà claire dans les modèles libéraux de démocratie délibérative (Benhabib, 1992). Plus encore, ajoutent les théoriciens agonistiques, il est nécessaire pour mettre en lumière les rapports de pouvoir et, du même coup, les " états consensuels » hégémoniques (Mouffe, 2010; Mouffe, 2013). C'est pourquoi le débat et le langage sont deux cibles de la gouvernementalité algorithmique selon Rouvroy.

Tout d'abord, dans la gouvernementalité algorithmique, le langage est " déprécié " en transformant la valeur de ce qui est communiqué. La valeur d'échange du langage supplante sa valeur d'usage, tirée du contenu (Ouellet, 2017, p. 5). En d'autres termes, le critère d'efficience devient celui de la circulation, plutôt que celui de la compréhension (Dean, 2005).

La valeur d'usage du langage est en effet subjective, tandis que la gouvernementalité algorithmique se présente plutôt comme " objective et immanente au monde lui-même, fonctionnant sur une logique purement inductive (statistique) ॥ (Rouvroy, 2012, s.p.). II est nécessaire, pour atteindre cette objectivité, de contourner, comme nous le mentionnons plus tôt, le sujet réflexif. Si cette réflexivité passe en partie par l'usage du langage et du débat, alors il devient nécessaire de formaliser le langage, de le « calculer » pour en retirer ses capacités subversives. Cet objectif pourrait être partiellement atteint, par exemple, en automatisant le débat, pour connaître « mieux que les personnes concernées elles-mêmes ce qu'elles pourraient faire ou vouloir » (Rouvroy, 2012, s.p.).

La régression du langage politique dans le contexte de la gouvernementalité algorithmique est en ce sens, selon Rouvroy, un enjeu d'importance. Le langage est dévitalisé, devient insignifiant : il y a «mépris du langage [et] de la polysémie " à cause du "positivisme enfermant» qui constitue la gouvernementalité algorithmique (Rouvroy, 2012, s.p.).

Or, peut-on vraiment craindre une actuelle, ou même future, dépolitisation du langage? L'idée d'automatiser les débats existe bel et bien, et est approfondie dans les recherches en intelligence artificielle, particulièrement dans la linguistique computationnelle. Le 
champ d'études particulier qui s'y attarde est celui de l'argumentation mining and processing.

\section{Argument mining/processing et gouvernementalité algorithmique}

Lorsqu'un gouvernement souhaite instaurer une politique publique, certains outils traditionnels sont mis en place: des consultations, des commissions publiques ou des sondages de groupes, par exemple. Pour chacune de ces méthodes, un certain niveau d'interaction humaine est nécessaire, soit à l'étape de la consultation, soit à celle de l'analyse. Or, dans une perspective d'efficacité, cela pose plusieurs problèmes : les personnes peuvent être incertaines, indécises, non informées, ou encore inconsistantes dans leurs réponses (Wyner et al., 2016). L'argument mining (AM) propose de résoudre, du moins en partie, ce problème.

Pour cette partie de la recherche, nous avons utilisé les actes des deux dernières "Conferences on Empirical Methods in Natural Language Processing " (EMNLP 2017 et 2018). Ces conférences, centrales dans les recherches en linguistique computationnelle et en intelligence artificielle, semblent être le point de convergence des dernières avancées scientifiques dans le domaine. Elles sont financées, sans grande surprise, par Apple, Facebook, Amazon et Google, entre autres (List of Sponsors, 2018). Différents ateliers s'y tiennent, par exemple sur l'apprentissage-machine du langage naturel ou sur les méthodes d'analyses computationnelles de la subjectivité et des sentiments, mais nous nous sommes concentrés sur l'atelier sur l'AM, qui en était en 2018 à sa cinquième édition (Workshops \& Co-located Events, 2018). Au total, trente-trois actes de conférence ont été explorés pour réussir à avoir un portrait global de l'état de la recherche en AM.

Cette exploration s'est faite à partir d'une analyse de contenu. Les textes ont été rassemblés dans le logiciel d'analyse textuelle Nvivo, pour plus facilement permettre d'en tirer les éléments pertinents, par exemple par la recherche de mots-clés ou l'observation de similarités entre les textes. Comme l'explique Paul Sabourin (2009, p. 416), l'analyse de contenu «a pour but de connaître la vie sociale à partir de cette dimension symbolique [le langage] des comportements humains ». Elle réfère donc à " un ensemble de démarches méthodologiques recourant à des méthodes et des techniques utilisées en vue d'interpréter des documents » (Sabourin, 2009, p. 416) dans l'objectif d'en connaître 
la signification sociale. L'analyse de contenu se déploie habituellement en cinq grandes étapes: la formulation de la question de recherche ou d'hypothèses; la sélection d'un échantillon représentatif; la définition des catégories d'analyses (inspirées du cadre théorique); le codage; et finalement le processus d'analyse (McMillan, 2000, p. 81-82). L'analyse présentée ici est en majeure partie qualitative et les textes, bien que lus et comparés attentivement, n'ont pas été soumis à un codage, puisque l'objectif était de tirer un portrait global du champ plutôt que d'étudier de façon isolée chacun des textes.

Nous avons choisi d'observer le champ d'études de l'AM, car il correspond à une partie de ce qui est entrevu comme futur " postpolitique » dans la conception de la gouvernementalité algorithmique de Rouvroy, c'est-à-dire l'existence d'un dispositif technologique en mesure de traiter le langage et le débat en tant que données et code et d'en retirer leur valeur politique.

\subsection{Argument mining : définition et objectifs}

L'AM est une forme avancée de compréhension du langage humain par une machine, qui est définie dans sa plus simple expression comme "la détection automatique de la structure argumentative d'un texte, la classification de ses composantes ainsi que de leurs relations argumentatives ${ }^{7}$ »(Moens, 2018, p. 2). Le texte analysé est initialement en langage naturel, c'est-à-dire en langage courant (Rocha et al., 2018).

Le principal objectif de l'AM est d'extraire automatiquement les arguments d'un texte ou d'un corpus de textes génériques pour les transformer en données structurées en mesure de faire fonctionner des " architectures d'argumentation et de raisonnement ${ }^{8}$ " (Lippi et Torroni, 2016, p. 2). Ces modèles fournissent à leur tour des structures algorithmiques "décisionnelles " (algorithmic decision making), qui exécutent des décisions de précisions et d'importances diverses à l'aide des connaissances générées (Yeung, 2018).

À partir d'une certaine base de données structurée (ou " knowledge base »), l'AM cherche à donner une représentation totale des connaissances disponibles et de leurs liens pour en tirer les « bonnes » conclusions. Wyner et al. (2016) développent par

${ }^{7}$ Citation originale: Argumentation mining has been defined as the automated detection of the argumentation structure and classification of both its component elements and their argumentative relationships.

${ }^{8}$ Citation originale : Computational models of argument and reasoning engines. 
exemple, à partir d'une base de connaissances annotée (literals and rules knowledge base, $L R K B$ ), une méthode grâce à laquelle il est possible de :

générer non seulement les arguments, mais aussi les contre-arguments, les contre-arguments de ces arguments et ainsi de suite. Une telle structure crée un graphique en arborescence, un " arbre d'argumentation », où les arguments sont des nœuds et les attaques à ces arguments sont les branches. Nous pouvons, à partir d'une base de données quelconque, générer tous les arguments et les contrearguments possibles ${ }^{9}$ (Wyner et al., 2016, p. 81).

L'AM contemple également, dans son processus, d'autres objectifs connexes. Par exemple, une analyse d'AM pourrait viser à déterminer automatiquement la qualité de la structure logique d'un argument (peu importe son contenu), ou encore à comprendre le raisonnement distinct derrière une argumentation donnée (Reisert et al., 2018, p. 80). Cette dernière méthode est nommée le Argument Reasoning Comprehension (ARC), et nécessite des « connaissances externes à propos du monde » (Botschen et al., 2018, p. 90).

Si l'AM reste un champ de recherche relativement récent qui trouve sa fertilité dans un contexte où les technologies de l'information sont ubiquitaires, les efforts pour pouvoir bâtir des systèmes automatisés complexes en mesure de repérer des éléments linguistiques en langage naturel pour procéder à leur classification datent de bien avant l'essor contemporain de l'intelligence artificielle. Dès le début des années 1960, on retrouve des recherches en "documentation automatique » qui cherchent entre autres à " extraire des textes [...] les unités linguistiques en mesure de représenter le contenu d'un document " (Salton, 1963, p. 440) pour bâtir des bases de données. Les recherches subséquentes mèneront par exemple à des méthodes de génération de langage naturel (Natural Language Generation ou $N G L$ ) susceptible de produire de la documentation technique de façon automatisée à partir de bases de données (Reiter et al., 1995). La relation entre bases de données et langage naturel prend bien évidemment aujourd'hui une importance particulière dans un

9 Citation originale: We can generate not only the arguments, but also the counterarguments, the counterarguments to these arguments (countercounterarguments), and so on recursively; such a structure is an argument tree, a graph where arguments are nodes and attack relations are (undifferentiated) arcs. From a given $L R K B$, we can generate all possible arguments and counter arguments. 
contexte d'informatisation croissante du social, mais les efforts en informatique pour s'attaquer à ce problème émergent plutôt de la première "vague » de l'intelligence artificielle, soit le modèle cognitiviste-symbolique qui dominera la deuxième moitié du $20^{\mathrm{e}}$ siècle (Cardon et al., 2018).

\subsection{Le problème de l'acquisition des connaissances}

Une difficulté importante, toutefois - dans le contexte qui nous intéresse -, est que ces méthodes ne sont pour l'instant pas en mesure de régler le " goulot d'étranglement des connaissances » (knowledge acquisition bottleneck), principal problème auquel fait face I'AM (Moens, 2018; Saint-Dizier, 2016; Wyner et al., 2016). C'est pourquoi, dans les expériences comme celle menée par Wyner et al., une certaine annotation humaine doit encore être effectuée.

Ce problème en est essentiellement un de traduction :

Le problème du goulot d'étranglement des connaissances est celui du passage de la forme en laquelle les personnes comprennent et expriment leurs connaissances vers une représentation formelle des connaissances qu'une machine peut traiter ${ }^{10}$ (Wyner et al., 2016, p. 70).

La difficulté est de transformer le langage humain, qui est culturel, signifiant, contextuel, polysémique, en un langage traitable de façon automatisée. Puisque ce qui nous intéresse ici est l'argument plus que le langage dans sa totalité, et que l'argument présuppose une certaine représentation du monde qui y est véhiculée, un autre problème se pose : celui de la « représentation des connaissances et du raisonnement sur les connaissances ${ }^{11}$ " (Wyner et al., 2016, p. 70). Ce problème n'est pas seulement celui de la compréhension par l'ordinateur du langage utilisé, dans toute sa complexité, mais celui de la compréhension de l'information qui porte une représentation du monde à l'intérieur même du langage (Wyner et al., 2016). En effet, pour qu'il y ait cohérence dans le langage, chaque " récepteur », qu'il soit humain ou ordinateur, doit posséder un « modèle mental » du langage en question, qui requiert toujours l'activation de grandes quantités de connaissances préalables précises ou génériques (Van Dijk, 2014, p. 250).

${ }^{10}$ Citation originale: The knowledge acquisition bottleneck is the problem of translating between the form in which participants know or express their knowledge and the formal representation of knowledge which a machine can process.

${ }^{11}$ Citation originale : Knowledge representation and reasoning (KR) problem. 
Pour le moment, expliquent Wyner et al. (2016), l'intervention humaine dans des contextes d'AM se révèle encore essentielle. Ces interventions sont effectuées par des "knowledge engineers » (la traduction française semble être " cogniticiens »), qui ont comme rôle d'intégrer dans le système informatique des connaissances spécifiques. Ce travail est interprétatif, autant sur le plan du contexte que de la sémantique, ce qui, pour les chercheurs en AM, pose un problème, car il y a possibilité de l'introduction d'une subjectivité humaine. En effet, ces cogniticiens basent implicitement leurs actions sur leurs connaissances linguistiques et usuelles, impliquant donc une certaine compréhension de la réalité.

Le problème de l'acquisition des connaissances par le langage est loin d'être récent. Lors de sa réflexion sur la lingua philosophica - le langage philosophique parfait capable de représenter l'ensemble du monde et des idées -, le philosophe et mathématicien Leibniz s'est buté à la même embûche, apparemment sans issue : la nécessité, pour arriver à ce " langage parfait ", de constituer une encyclopedia universalis, où la totalité des concepts et de leurs relations devait être incluse (Kluge, 1980). Un autre philosophe du langage, Frege, exprimait plutôt l'idée selon laquelle il était possible d'arriver à un " langage parfait », " un système symbolique artificiel conçu pour refléter la structure de la réalité ainsi que pour emmener clairvoyance et complétude dans le raisonnement ${ }^{12}$ » (Kluge, 1980, p. 141), sans nécessairement passer par l'encyclopedia universalis, si le langage était en mesure par lui-même de remplacer la logique comme activité réflexive.

Bref, le rapport entre connaissance, langage et automatisation est fort, mais reste à approfondir. La difficulté de l'AM à passer d'un texte écrit par un humain sans structure argumentative claire (un long commentaire sur Facebook, par exemple) à une structure de données traitable par un ordinateur réside en partie dans un autre problème qui transcende les recherches en intelligence artificielle : celui du passage du langage naturel au langage formel (Cabrio et Villata, 2018).

La conception philosophique des termes «naturel » (naturalis) et "formel» (formalis), conception qui est reprise dans la compréhension linguistique des concepts «langage naturel » et "langage formel», semble provenir principalement de l'école aristotélicienne et de la scolastique, particulièrement des enseignements de Thomas d'Aquin au $13^{\mathrm{e}}$ siècle (De Wulf, 1899;

${ }^{12}$ Citation originale: An artificial symbolic system designed to reflect the structure of reality and intended to effect clarity and completeness in reasoning. 
Van Steenberghen, 1985). Le naturalis est exploré dans la philosophie théorique, tandis que le formalis est exploré dans la logique. Plusieurs grands penseurs du langage proviennent d'ailleurs de la tradition de la logique philosophique, dont notamment Russell, Frege et Wittgenstein. C'est Frege, plus précisément, à qui on doit la notion de « langage formel » (ainsi que la première tentative d'en créer un), dans son livre "Begriffsschrift », publié en 1879. Frege souhaitait, comme Leibniz avant lui, créer un langage « logiquement parfait » (Kluge, 1980).

Un langage formel, à la base, est un système logique - certains le réduisent même à un système mathématique (Hopcroft et Ullman, 1969) - constitué d'un ensemble de symboles appelé simplement " alphabet » (Wintner, 2010). Le langage formel doit être constitué d'une grammaire et d'une syntaxe qui ne présentent aucune ambiguïté, c'est-à-dire qu'une phrase (généralement découpée en "phrase-verbe » et "phrase-nom ») ne peut avoir qu'un sens unique et ne doit contenir aucune redondance. Ces caractéristiques rendent difficile le passage fidèle du langage naturel au langage formel. La conception derrière la notion de langage formel, tel que brièvement mentionné plus tôt, est qu'il existe une structure analysable de la réalité, structure qu'il est possible de réduire en un certain nombre d'unités de base et de relations (Kluge, 1980). Si cette conception s'avère exacte, alors il serait possible, à partir d'un choix calculé de symboles "de base » et d'axes, de dériver l'ensemble des représentations possibles de la réalité.

Le passage du langage naturel au langage formel est nécessaire à l'AM (et à la plupart des champs de recherche en intelligence artificielle qui nécessitent du traitement de langage), tout en étant le reflet politiquement incarné de l'AM elle-même. La formalisation du langage et la formalisation de l'argumentation procèdent toutes les deux de la même logique : réduire une forme discursive à un certain nombre d'unités de base analysables; faire disparaître de ces unités toute possibilité de polysémie, d'ambiguïté, de subversivité; et ensuite, traiter mathématiquement le discours, faisant disparaître du même coup ses fonctions subjectives et conséquemment subversives.

\subsection{Champs d'applications}

Les recherches en $A M$, selon notre échantillonnage mené avec les actes de conférence du EMNLP, semblent avoir plusieurs champs d'applications. Quelques-unes des recherches présentées 
se concentraient sur l'AM dans le domaine scientifique (surtout en biologie et en médecine), dans l'objectif de pouvoir résumer certains débats. D'autres se situaient plutôt dans le domaine du droit, dans l'objectif de bâtir automatiquement des arguments légaux à partir de textes de loi. Quelques autres, plus isolées, portaient par exemple sur les systèmes de recommandation d'Amazon ou sur le link prediction (la prédiction automatisée de nouveaux liens dans des réseaux, par exemple de nouvelles amitiés Facebook). Les recherches restantes portaient sur les méthodes générales de l'AM sans les appliquer à un champ particulier.

Le champ d'applications de l'AM qui nous intéresse principalement est bien sûr celui relatif au débat politique. Nous avons constaté que plus du tiers des recherches portait directement sur ce thème (12 sur 33 ). Voici quelques exemples qui nous ont paru pertinents, sans nommer toutes les recherches présentées :

- Un des textes proposait une méthode pour évaluer automatiquement le niveau de persuasion des interventions dans un fil de commentaires (online comments convincingness evaluation) (Gu et al., 2018);

- Un des textes proposait une méthode pour analyser et prédire automatiquement les arguments et le langage des campagnes de relations publiques dans un contexte de défense de réputation (predict the language of reputation defence) (Naderi et Graeme, 2018);

- Un autre proposait un agent de dialogue argumentatif en mesure de débattre avec des personnes humaines sur des sujets complexes et controversés, agent nommé Dave the Debater (Thu Le et al., 2018);

- Un article proposait une méthode d'AM pour générer automatiquement les prémisses et les conclusions d'une argumentation sur un thème précis, en intégrant des connaissances contextuelles et une compréhension plus large des sujets connexes, un défi dans l'AM (Lawrence et Reed, 2017a);

- Les mêmes chercheurs proposaient, dans une autre conférence, une technique d'AM (Complex Argumentative Interaction (CAI)) pour reconstruire de façon formalisée la structure argumentative d'un débat mené à large échelle, par exemple celui des élections présidentielles américaines de 2016, utilisé comme corpus (Lawrence et Reed, 2017b); 
- Un dernier texte classifiait et organisait plus de 200000 arguments politiques récoltés sur Internet concernant la possibilité d'une nouvelle constitution au Chili (Fierro et al., 2017).

\subsection{Automatisation de l'argumentation politique}

Bien que tirer automatiquement les arguments et leurs contrearguments d'une source en langage naturel demeure un défi, les progrès récents dans le champ de l'AM sont significatifs (Wyner et al., 2016, p. 73). Selon le corpus évalué, des progrès rapides sont faits dans plusieurs champs de recherche du domaine :

Le champ de l'argument mining est en croissance rapide grâce à trois principaux phénomènes: premièrement, le succès académique et commercial des méthodes d'opinion mining et d'analyse des sentiments, sur lesquelles se base l'argument mining; deuxièmement, une forte demande commerciale de la part d'entreprises telles qu'lBM; troisièmement, le développement des infrastructures, des outils, et de la compréhension théorique des structures argumentatives autant dans le monologue que le dialogue ${ }^{13}$ (Lawrence et Reed, 2017a, p. 39).

Présumer une réussite complète et totale du projet de l'AM serait faire l'erreur du déterminisme. II nous paraît tout de même pertinent de réfléchir en amont à la perspective de l'argumentation automatisée, qui serait bien intégrée dans le contexte du postpolitique et de la gouvernementalité algorithmique explicité plus tôt. Les auteurs du texte "Toward Artificial Argumentation », dans le "Al Magazine », écrivent au tout début de leur texte: "Les humains argumentent. Cette caractéristique distinctive est à la fois une capacité cognitive importante et un puissant phénomène social ${ }^{14}$ » (Atkinson et al., 2017, p. 25). Comment penser cette capacité cognitive unique et ce puissant constituant social dans un contexte où l'argumentation serait retirée de sa spécificité humaine?

Une telle perspective sur l'argumentation et le débat évacue en bonne partie le politique, tout d'abord par l'action de numérisation

\footnotetext{
13 Citation originale: Argument mining has recently been enjoying rapid growth, propelled by three drivers: first, the academic and commercial success of opinion mining and sentiment analysis techniques upon which argument mining builds; second, a strong commercial appetite for such technologies from companies such as IBM; and third, the development of infrastructure and tools for and theoretical understanding of argument structure in both monologue and dialogue.

${ }^{14}$ Citation originale: Humans argue. This distinctive feature is at the same time an important cognitive capacity and a powerful social phenomenon.
} 
du langage et des structures argumentatives qui est dépolitisante en elle-même, comme le montrent Rouvroy et Berns (Rouvroy, 2012; Rouvroy et Berns, 2013). En effet, un modèle d'AM qui serait en mesure de mathématiser, résumer, restructurer des arguments dans un débat pour en tirer automatiquement une conclusion algorithmiquement bâtie ne pourrait faire autrement que de présenter une conclusion aseptisée, fidèle au cadre politique déjà en place, de la même façon que la statistique ne considère pas les données aberrantes. Or, même si elles ne sont pas considérées, ces données existent tout de même : d'aucune façon les méthodes de traitement automatisé du débat ne parviendraient à éliminer entièrement les antagonismes, qui sont, comme nous l'avons observé plus tôt grâce à Mouffe (2010), la condition même de l'exercice du politique et de l'existence de la démocratie.

Notre corpus d'analyse est certes limité, mais nous pouvons tout de même constater un intérêt particulier pour le domaine du politique et de l'administration publique dans les recherches en AM. Être en mesure d'analyser automatiquement de grandes quantités de textes argumentatifs - sous toutes leurs formes, c'est-à-dire être en mesure d'analyser automatiquement l'expression politique humaine langagière - est un projet qui semble, pour ce champ, porteur. Un tel outil permettrait de prendre des décisions plus rapides et plus efficaces, de combiner tous les arguments avancés sur un graphique réduit, d'éviter de collectivement " perdre du temps " dans de longues discussions qui mènent de toute façon à des conclusions qu'il serait possible de prédire.

Même si l'objectif semble similaire, transformer le débat et son langage en données et en graphes a des implications plus grandes que, disons, traiter de façon algorithmique des données pour arriver à la configuration optimale d'un réseau d'aqueducs. Le langage est source de connaissances (Van Dijk, 2014), d'identités (Carroll et al., 2008), de pouvoir (Bourdieu, 1977), d'affects (Deleuze et Guattari, 2005) et d'idéologies (Thompson, 1987). Surtout, le langage dépasse aujourd'hui grandement ce qu'il est possible de traiter de façon informatique : tant que l'informatique ne pourra pas exécuter un passage du langage naturel au langage formel qui serait parfait, elle ne pourra pas dépolitiser le langage. II nous semble tout de même que des champs comme celui de l'argumentation mining doivent être analysés de façon critique, holistique et approfondie. Le langage est en effet un aspect peu exploité de la gouvernementalité algorithmique. II constitue toutefois pour Rouvroy un espace commun prospère " pour une critique, une récalcitrance, et de 
nouveaux modes d'individuation face à la gouvernementalité algorithmique " (Rouvroy, 2012, s.p.). Elle-même n'y revient d'ailleurs que brièvement dans ses publications subséquentes (Rouvroy, 2016; Rouvroy et Berns, 2013; Rouvroy et Stiegler, 2015).

Malgré les avancées dans le traitement du langage naturel, plus particulièrement en argumentation automatisée, la dépolitisation et la dépréciation du langage craintes par Rouvroy et Berns ne seraient selon nous envisageables aujourd'hui que dans un contexte où l'informatique arriverait à faire une ontologie complète du monde et du langage. C'est pourquoi, comme le mentionne d'ailleurs Rouvroy, qui voit tout de même dans le langage un lieu de résistance possible à la gouvernementalité algorithmique, le langage reste une " ligne de fuite » :

II s'agira de montrer que l'enjeu fondamental - ce qu'il y aurait à sauver comme ressource antécédante [sic] à tout "sujet », comme " excès " du monde sur la réalité numérique - est le commun, cet entre, ce lieu de comparution dans lequel nous sommes adressés les uns aux autres. Le mode d'adresse qui nous relie est essentiellement langagier. Le langage est "forme " qui est tout à la fois ligne de fuite polysémique / polyphonique, principe de liaison (« faire tenir ensemble »), projection miroitante, motifs d'advenirs évoqués. Cette « forme » (esthétique, en tant qu'elle est rapport affectif entre existants), le langage (si l'on accepte d'en libérer toutes les puissances poétiques), est à mille lieues de toute personologie [sic]. II est la forme impersonnelle par excellence, manifestation de l'excès du monde sur le réel (Rouvroy, 2012, s.p.).

\section{Conclusion: perspectives post-politiques de} l'argumentation automatisée

Le projet post-politique tel que présenté au début de ce texte trouve donc de forts échos dans le champ de recherche qu'est l'AM. II en va de même pour le contexte de la gouvernementalité algorithmique tel que vu par Rouvroy et Berns.

Nous avons tenté, dans ce texte, de mener un exercice de réflexion théorique sur les liens entre la gouvernementalité algorithmique, le langage et le post-politique. Pour ce faire, nous avons exploré de façon critique le champ d'études et de recherche sur l'argumentation mining, une méthode qui, dans son idéal, arriverait à transformer automatiquement l'activité argumentative 
humaine en données numérisables et "computables" pour générer des politiques et des décisions. Nous avons voulu montrer comment l'AM tente d'éliminer du débat les possibilités d'antagonisme et d'éliminer du langage les possibilités de résistance, mais se bute à des problèmes paradigmatiques et épistémologiques qui restent sans réponse.

Cette réflexion est certes à un stade précoce, et présente plusieurs limites, dont un corpus restreint et une compréhension tout de même limitée des méthodes informatiques qui soutiennent les procédés d'AM. Nous souhaitions principalement poser un regard critique sur le champ de recherche de l'AM, explorer ses fonctions et ses objectifs, et l'analyser dans un contexte particulier, soit celui du post-politique et de la gouvernementalité algorithmique. Les liens restent à éclaircir et à approfondir à plusieurs niveaux, mais sont tout de même incontournables, à notre sens, et méritent d'être analysés de façon plus précise.

En effet, la force des relations entre les technologies de l'information et le politique est aujourd'hui telle que le développement de l'AM comme bien d'autres champs de l'informatique, d'ailleurs, développés avant que l'on comprenne et même que l'on anticipe leurs conséquences - pourrait se révéler problématique. Même si, comme mentionné, l'idée d'un système automatisé en mesure d'abstraire et de saisir le langage dans son entièreté nous paraît peu probable, il n'en reste pas moins que la mise en place de méthodes tirées de l'AM pour introduire dans des débats publics des agents automatisés, comme c'est déjà le cas avec les bots politiques, peut avoir de graves conséquences. Chavalarias (2012) montre bien comment l'accès à un nombre restreint de données comportementales dans un réseau permet de prédire avec une grande précision le comportement des membres du réseau dans leur ensemble. II découle de cette constatation une conséquence, soit la facilité - pour des acteurs en mesure de le faire - de modifier le comportement d'une masse à partir de "nudges " minimaux. À cet effet, l'étude de 2014 de Kramer et al. est frappante. Menée sur près de 700000 utilisateurs de Facebook (à leur insu), elle montre qu'il est possible d'influencer l'état émotionnel des utilisateurs en les exposant à un lexique langagier soit négatif, soit positif, un phénomène désigné dans la recherche comme de la "contagion émotionnelle ». Une attention particulière doit être portée aux techniques qui cherchent à réduire la friction entre langage formel et langage naturel, particulièrement lorsque celles-ci concernent une fonction sociale essentielle du langage, soit l'échange politique, 
sans quoi les conséquences de cette gouvernementalité algorithmique s'en trouveront aggravées. 


\section{Références}

Atkinson, K. et al. (2017). Towards Artificial Argumentation. Al Magazine, 38(3), 25-36. https://doi.org/10.1609/aimag.v38i3.2704

Beck, U. (1992). Risk Society : Towards a New Modernity. Londres, Royaume-Uni : Sage.

Benhabib, S. (1992). Models of Public Space: Hannah Arendt, the Liberal Tradition, and Jurgen Habermas. Dans C. J. Calhoun (dir.), Habermas and the Public Sphere (p. 99-108). Cambridge, États-Unis (MA) : MIT Press.

Besnier, J.-M. (2013). Quelles utopies à l'ère du numérique? Études, 19(7), 43-51.

Bonenfant, M., Ménard, M., Mondoux, A. et Ouellet, M. (2015). Big Data, médiation symbolique et gouvernementalité. Dans E. Broudoux \& G. Chartron (dir.), Open Data - Big Data: Quelles valeurs? Quels enjeux? Actes du colloque "Document numérique et société " (p. 31-42). Paris, France : De Boeck Supérieur.

Botschen, T., Sorokin, D. et Gurevych, I. (2018). Frame- and EntityBased Knowledge for Common-Sense Argumentative Reasoning. Proceedings of the 5th Workshop on Argument Mining, 90-96.

Bourdieu, P. (1977). Sur le pouvoir symbolique. Annales, 32(3), 405-411. doi : https://doi.org/10.3406/ahess.1977.293828

Cabrio, E. et Villata, S. (2018). Five Years of Argument Mining: A Data-driven Analysis. Proceedings of the Twenty-Seventh International Joint Conference on Artificial Intelligence, 5427-5433. doi : https://doi.org/10.24963/ijcai.2018/766

Cardon, D., Cointet, J.-P. et Mazières, A. (2018). La revanche des neurones: L'invention des machines inductives et la controverse de l'intelligence artificielle. Réseaux, 5(11), 173-220.

Carroll, S., Motha, S. et Price, J. N. (2008). Accessing Imagined Communities and Reinscribing Regimes of Truth. Critical Inquiry in Language Studies, 5(3), 165-191. doi : https://doi.org/10.1080/15427580802285704 
Chavalarias, D. (2012). La société recommandée : De la conjecture de von Foerster aux sciences sociales prédictives. Dans P.A. Chardel, B. Reber, \& C. Gossart, Conflits des interprétations dans la société de l'information: Éthique et politique de l'environnement (p. 41-56). Paris, France: Lavoisier. https://doi.org/10.1177/2053951715621086

Cossette, S. (2018). Algorithmique, microtargeting et discours politique: Automatisation du discours politique et élections présidentielles américaines de 2016 (mémoire de maîtrise). Université du Québec à Montréal, Canada. https://archipel.uqam.ca/12463/1/M15946.pdf

Dardot, P. et Laval, C. (2010). Néolibéralisme et subjectivation capitaliste. Cités, 41 35-50. doi : https://doi.org/10.3917/cite.041.0035

De Wulf, M. (1899). La synthèse scolastique. Revue philosophique de Louvain, 6(21), 41-65. doi : https://doi.org/10.3406/phlou.1899.1642

Dean, J. (2005). Communicative capitalism: Circulation and the foreclosure of politics. Cultural Politics, 1(1), 51-74.

Deleuze, G. et Guattari, F. (2005). Qu'est-ce que la philosophie? Paris, France : Les Éditions de Minuit.

Fierro, C., Pérez, J. et Quezada, M. (2017). 200K+ Crowdsourced Political Arguments for a New Chilean Constitution. Proceedings of the 4th Workshop on Argument Mining, 11-21.

Fukuyama, F. (1992). The End of History and the Last Man. New York, États-Unis (NY): Free Press.

Giddens, A. (1994). Beyond Left and Right: The Future of Radicals Politics. Cambridge, Royaume-Uni: Polity Press.

Giddens, A. (1998). The Renewal of Social Democracy. Cambridge, Royaume-Uni: Polity Press.

Gu, Y., Wei, Z., Xu, M., Fu, H., Liu, Y. et Huang, X. (2018). Incorporating Topic Aspects for Online Comment Convincingness Evaluation. Proceedings of the 5th Workshop on Argument Mining, 97-104. 
Hopcroft, J. E. et Ullman, J. D. (1969). Formal Languages and Their Relation to Automata. Boston, États-Unis (MA) : AddisonWesley Longman Publishing Co., Inc.

Kluge, E.-H. W. (1980). Frege, Leibniz and the Notion of an Ideal Language. Studia Leibnitiana, 12(1), 140-154.

Kramer, A. D. I., Guillory, J. E. et Hancock, J. T. (2014). Experimental evidence of massive-scale emotional contagion through social networks. Proceedings of the National Academy of Sciences, 111(24), 8788-8790. doi : https://doi.org/10.1073/pnas.1320040111

Lasswell, H. D. (1955). Current studies of the decision process: Automation versus creativity. Western Political Quarterly, 8(3), 381-399.

Lawrence, J. et Reed, C. (2017a). Mining Argumentative Structure from Natural Language text using Automatically Generated Premise-Conclusion Topic Models. Proceedings of the 4th Workshop on Argument Mining, 39-49.

Lawrence, J. et Reed, C. (2017b). Using Complex Argumentative Interactions to Reconstruct the Argumentative Structure of Large-Scale Debates. Proceedings of the 4th Workshop on Argument Mining, 108-117.

Lippi, M. et Torroni, P. (2016). Argumentation Mining: State of the Art and Emerging Trends. ACM Trans. Internet Technol., 16(2), 10:1-10:25. doi : https://doi.org/10.1145/2850417

List of Sponsors. (2018). https://emnlp2018.org/sponsors/list/

McMillan, S. J. (2000). The microscope and the moving target: The challenge of applying content analysis to the World Wide Web. Journalism and Mass Communication Quarterly, 77(1), 80-98.

Moens, M.-F. (2018). Argumentation mining: How can a machine acquire common sense and world knowledge? Argument \& Computation, 9(1), 1-14. doi : https://doi.org/10.3233/AAC170025

Mouffe, C. (2010). Politique et agonisme. Rue Descartes, (67), 18-24.

Mouffe, C. (2013). Agonistics: Thinking the world politically. Londres, Royaume-Uni : Verso. 
Naderi, N. et Graeme, H. (2018). Using context to identify the language of face-saving. Proceedings of the 5th Workshop on Argument Mining, 111-120.

Ouellet, M. (2017). L'Empire de la communication: Post-vérité et post-politique dans la postmodernité. Revue Liberté, (318).

Ouellet, M., Bonenfant, M., Ménard, M. et Mondoux, A. (2016). Big Data, gouvernementalité et industrialisation des médiations symboliques et politico-institutionnelles. Interfaces numériques, 5(2), 147-170.

Reisert, P., Inoue, N., Kuribayashi, T. et Inui, K. (2018). Feasible Annotation Scheme for Capturing Policy Argument Reasoning using Argument Templates. Proceedings of the 5th Workshop on Argument Mining, 79-89.

Reiter, E., Mellish, C. et Levine, J. (1995). Automatic Generation of Technical Documentation. Applied Artificial Intelligence, 9(3), 259-287. doi : https://doi.org/10.1080/08839519508945476

Rocha, G., Stab, C., Lopes Cardoso, H. et Gurevych, I. (2018). Cross-Lingual Argumentative Relation Identification: From English to Portuguese. Proceedings of the 5th Workshop on Argument Mining, 90-96.

Rouvroy, A. (2012). Mise en (n)ombres de la vie même: Face à la gouvernementalité algorithmique, repenser le sujet comme puissance. Mediapart. https://blogs.mediapart.fr/antoinetterouvroy/blog/270812/mise-en-nombres-de-la-vie-memeface-la-gouvernementalite-algorithmique-repenser-le-sujetcom

Rouvroy, A. (2016). La gouvernementalité algorithmique: Radicalisation et stratégie immunitaire du capitalisme et du néolibéralisme? La Deleuziana, (3), 30-36.

Rouvroy, A. et Berns, T. (2013). Gouvernementalité algorithmique et perspectives d'émancipation. Réseaux, (177), 163-196. doi : https://doi.org/10.3917/res.177.0163

Rouvroy, A. et Stiegler, B. (2015). Le régime de vérité numérique. Socio, (4), 113-140. doi : https://doi.org/10.4000/socio.1251

Sabourin, P. (2009). L'analyse de contenu. Dans B. Gauthier (dir.), Recherche sociale: De la problématique à la collecte des 
données (p. 415-444). Québec, Canada: Presses de l'Université du Québec.

Saint-Dizier, P. (2016). Challenges of Argument Mining: Generating an Argument Synthesis based on the Qualia Structure. The 9th International Natural Language Generation conference: Actes du colloque tenu à l'université Heriot-Watt du 5 au 8 septembre 2016 à Édimbourg (p. 79-83). Édimbourg, Royaume-Uni : Association for Computational Linguistics. doi : https://doi.org/10.18653/v1/W16-6613

Salton, G. (1963). Associative Document Retrieval Techniques Using Bibliographic Information. Journal of the ACM, 10(14), 440-457.

Thompson, J. B. (1987). Langage et idéologie. Langage et société, 39(1), 7-30. doi : https://doi.org/10.3406/lsoc.1987.2340

Thu Le, D., Nguyen, C.-T., Nguyen, K. A. et Inui, K. (2018). Dave the debater: A retrieval-based and generative argumentative dialogue agent. Proceedings of the 5th Workshop on Argument Mining, 121-131.

Van Dijk, T. A. (2014). Discourse and Knowledge: A Sociocognitive Approach. Cambridge, Royaume-Uni: Cambridge University Press.

Van Steenberghen, F. (1985). La structure de la philosophie théorique selon $\mathrm{S}$. Thomas d'Aquin. Revue philosophique de Louvain, 83(60), 536-558.

Wintner, S. (2010). Formal Language Theory. Dans A. Clark, C. Fox, et S. Lappin (dir.), The Handbook of Computational Linguistics and Natural Language Processing (p. 11-42). Chichester, Royaume-Uni: Wiley-Blackwell. doi: https://doi.org/10.1002/9781444324044

Workshops \& Co-located Events. https://emnlp2018.org/program/workshops/

(2018).

Wyner, A., van Engers, T. et Hunter, A. (2016). Working on the argument pipeline: Through flow issues between natural language argument, instantiated arguments, and argumentation frameworks. Argument \& Computation, 7(1), 69-89. doi : https://doi.org/10.3233/AAC-160002 
Yeung, K. (2018). Algorithmic regulation: A critical interrogation. Regulation \& Governance, 12(4), 505-523. doi : https://doi.org/10.1111/rego.12158

Zizek, S. (2005). Against Human Rights. New Left Review (34), 115131. 